\title{
COMUNICACIONENLOSTIEMPOSDELACOLERA: CLNICOSYRADIOLOGOS
}

\section{Drs. César García M(1), Dulia Ortega $T^{(2)}$.}

1. Servicio de Radiología. Clínica Alemana de Santiago

2. Centro de Imagenología. Hospital Clínico Universidad de Chile

\begin{abstract}
We have more than enough evidence pointing that effective clinical communication among specialists is not as good as we think. Undoubtedly, it is not a minor issue: it is directly related to patient outcomes. Radiologists are not excluded of these problems; they are part of clinical communication. Exams results must be delivered to referring physicians promptly and effectively. Coping with these issues, a primary consideration is to consider effective clinical communication a standard of good radiology practice included in regulatory standards developed for this purpose. Radiologist-to-radiologist communication is part of this matter and must be ruled by ethical, professional and respect considerations, specially referred to diagnostic error communication. The goals of these efforts are to accomplish properly our most important duty: good radiological care to our patients. Key words: Audit, Clinico-radiologic conferences, Diagnostic performance, Radiology and radiologists.
\end{abstract}

Resumen: Existen elementos suficientes que nos indican que la comunicación efectiva entre los especialistas clínicos, incluyendo los de apoyo, no es toda lo buena que debiera ser y no sólo es un asunto de buena educación y consideración hacia otros colegas, sino que se relaciona directamente con los resultados de la atención médica, la cual puede ser seriamente afectada por fallas en la transmisión y comunicación de datos clínicos, resultados de exámenes, diagnósticos o terapéuticos. Los radiólogos no están exentos de estos problemas ni de sus consecuencias, por ello es importante considerar la comunicación oportuna con los tratantes como un objetivo de buena atención y debería ser normado en estándares que sugieran conductas para una comunicación apropiada y oportuna del resultado de los exámenes. Del mismo modo, la comunicación con

García C, Ortega D. Comunicación en los tiempos de la colera: Clinicos y radiólogos. Rev Chil Radiol 2004; 10:187-191.

Correspondencia: Dr. César García M. cgarcia@alemana.cl nuestros pares radiólogos, especialmente del error diagnóstico, debe conducirse en un marco ético, con respeto y profesionalismo. Todo ello con el fin de obtener nuestro objetivo único: la buena atención de nuestros pacientes.

Palabras clave: Auditorías, Conferencias clínicoradiológicas, Desempeño diagnóstico, Radiología y radiólogos.

\section{Introducción}

La comunicación entre los médicos clínicos y los de especialidades de apoyo suele ser un desastre $\mathrm{y}$ esto parece ser un problema universal, independiente de las personas, del tipo de sistema de atención involucrado y de la especialidad médica que actúa.

El tipo de cultura organizacional, que hace posible esta mala comunicación, ha sido abordado en países anglosajones en informes gubernamentales y trabajos independientes que coinciden en su diagnóstico y en la naturaleza de sus propuestas. El mejoramiento de la comunicación clínica tiene como objetivo aumentar la calidad y eficiencia de la atención médica y reducir la ocurrencia de eventos adversos en su práctica. En nuestro país, no conocemos estudios que aborden el tema, pero en forma empírica podemos deducir que el problema existe, a lo menos en cierto grado.

Una comunicación escasa, tardía y mal conducida, además de una pérdida de tiempo, es una amenaza a la atención del paciente y es la principal causa de los errores evitables, que pueden conducir a daños e incluso muerte ${ }^{(1,2)}$.

La revisión de la literatura a nuestro alcance, nos revela que las inquietudes por el análisis del tema partió de los médicos clínicos debido a su importancia en los resultados de la atención médica integral ${ }^{(3)}$. La gran mayoría de los autores coincide que para lograr mejorías efectivas en la comunicación clínica, los médicos requieren, sistemas más estructurados para el acopio de datos, un entrenamiento y adecuación a las nuevas tecnologías de la información, que emergieron en la década pasada, como herramientas llenas de promesas para lograr una mejor calidad de atención y cuyo impacto real está recién siendo evaluado $^{(4)}$. 


\section{Situación en radiología}

La práctica de la radiología y de quienes la ejercemos no esta ajena a este problema. En un articulo previo, revisamos la comunicación entre pacientes y radiólogos en el marco del ejercicio actual de la radiología y discutimos algunas medidas prácticas para mejorarla haciéndola más efectiva y humanizada ${ }^{(5)} \mathrm{El}$ objetivo actual es analizar la comunicación de los radiólogos con los médicos tratantes y con nuestros pares radiólogos, para optimizar la calidad de la atención a los pacientes.

Lejos de constituir tan solo una especie de decálogo de buenas maneras, la comunicación clínica tiene como resultado calidad de atención. Para clarificar este punto mencionaremos algunas acciones que lo ejemplifican: continuidad de la atención, disponibilidad de los datos clínicos pertinentes, oportunidad de la obtención de los resultados por el clínico, en especial si estos son urgentes o inesperados, etc ${ }^{(6)}$.

La manera en que es efectuada la comunicación en radiología se puede clasificar en dos grandes grupos: 1) La comunicación escrita o informe radiológico que acompaña a las imágenes obtenidas para cada paciente. 2) La comunicación verbal directa, personal o telefónica. El correo electrónico y el fax pueden ser considerados como comunicación escrita, pero tienen el riesgo de no ser leídos oportunamente. La comunicación escrita por medio del informe radiológico ha sido objeto de una publicación anterior ${ }^{(7)}$.

E1 posicionamiento de los pacientes como consumidores de planes de salud y la elevada conciencia acerca de sus derechos, la incorporación de nuevas tecnologías y de técnicas intervencionales e invasivas han hecho que el ejercicio de la radiología haya sufrido un cambio radical en los últimos 20 años. Ha habido una integración a equipos multidisciplinarios en prácticamente todas las especialidades, con participación activa en el manejo de los pacientes, ya sea con opinión experta en imágenes o a través de intervencionismo terapéutico. Hemos asumido, por tanto una mayor exposición a reclamos y demandas de pacientes insatisfechos. Paradójicamente, asistimos a una mejoría en la satisfacción del ejercicio de la especialidad $^{(8)}$.

La comunicación entre clínicos y radiólogos sufrió un cambio con el advenimiento de las tecnologías seccionales las que por su naturaleza, al ampliar el rango de diagnóstico y complejidad de las imágenes requirió de una mayor cuota de intercambio de información, tanto al momento de obtener o informar los exámenes, como en las reuniones clínicas.

Hasta aquí, todo resulta idílico, pero en la práctica diaria, a veces, el panorama es desastroso: clínicos disconformes con los diagnósticos radiológicos o por la ausencia de comunicación directa de hallazgos urgentes, pacientes que reclaman a sus médicos tratantes por la manera como «su lumbrera me trató durante el examen» o comentarios que resultan ofensivos para el paciente en el informe radiológico, etc. Esto a su vez genera, a lo menos, insatisfacción en el médico tratante por la forma en que su paciente fue atendido, y en el paciente por el trato recibido.

Aún la comunicación más rutinaria y amistosa de los radiólogos con los médicos tratantes consume tiempo de trabajo, un bien escaso para ambos grupos. Sin embargo es una obligación ética y legal que no podemos eludir. Ha sido demostrado que las entrevistas entre clínicos y radiólogos pese a consumir tiempo, son de claro beneficio para ayudar a precisar diagnósticos, alterar decisiones terapéuticas e incluso orientar a otros diagnostico al sugerir exámenes de imágenes complementarios ${ }^{(9)}$.

Un dilema que se presenta es la comunicación directa, por parte del radiólogo, del resultado de los exámenes al paciente. En ausencia de normas acordadas con los tratantes, el radiólogo no puede eludir la responsabilidad de comunicar resultados a pacientes que lo solicitan, sin olvidar nunca que es el médico tratante quien una vez hecho el diagnóstico informa al paciente y propone las alternativas terapéuticas.

La pregunta resultante es ¿Hasta donde llega la responsabilidad del radiólogo para comunicar los hallazgos directamente al paciente? ${ }^{(10)}$

Surgen aquí varios matices. La comunicación directa de hallazgos urgentes o inesperados al paciente al no poder ubicar al médico tratante está asentada en normas, pero no libera al radiólogo de la responsabilidad de comunicarse con este último. Los desacuerdos comienzan cuando, en áreas como la mamografía, encontramos un hallazgo que debemos controlar de acuerdo a la clasificación de BI-RADS y la paciente desaparece de los controles, no vuelve al médico tratante y reaparece con un cáncer invasor. ¿Debe el radiólogo seguir a todas sus pacientes con hallazgos sospechosos para controlarlos? ¿Acaso el médico tratante no es el primer responsable de su paciente y debe preocuparse de ver las imágenes que el mismo solicitó y aplicar el principio de la continuidad de la atención? ${ }^{(11,12)}$

\section{Estándares de comunicación}

Una forma de solución es la elaboración de normas y protocolos cuyo conjunto conforman los estándares de comunicación. El uso y conocimiento de estas normativas optimiza el manejo de los pacientes y tiene como factor agregado, el reducir los riesgos de responsabilidad profesional médica.

Aunque tradicionalmente los radiólogos muchas veces no tienen contacto personal con los pacientes, existen de todos modos los deberes y obligaciones de toda relación médico-paciente, incluida la comunicación efectiva con estos y con los médicos tratantes.

Clásicamente, los radiólogos enviamos, a los médicos que solicitan un examen, un informe escrito 
que contiene hallazgos e interpretación de las imágenes que se anexan. Para cumplir con la obligación de practicar la atención debida, debemos coordinar nuestros esfuerzos con el médico tratante para que los hallazgos anormales sean puestos en su conocimiento en forma eficiente y oportuna de modo de favorecer la atención óptima del paciente.

Los estándares de comunicación desarrollados, por el "American College of Radiology" (ACR) y por la "Canadian Association of Radiologists" (CAR), delimitan y definen las preocupaciones de los radiólogos sobre los métodos apropiados de comunicación.

El estándar aconseja, no obliga, a los radiólogos para coordinar sus esfuerzos con los del médico tratante en orden a obtener una mejor atención. Esto requiere o puede necesitar en algunas circunstancias de comunicación directa de los hallazgos inusuales, inesperados o urgentes al médico antes de tener el informe escrito definitivo. En tales circunstancias, el radiólogo o su representante deberían intentar comunicarse directamente (en persona o por teléfono) con el médico tratante o su representante. La oportunidad de la comunicación directa debería basarse en la inmediatez de la situación crítica. Tal es el caso de hallazgos que ponen en riesgo de morbilidad o mortalidad aguda y necesitan un manejo terapéutico inmediato: neumotórax a tensión, pericarditis constrictiva, apendicitis aguda. También, la detección de probables enfermedades con morbilidad o mortalidad suficientemente grave, pero no aguda, que necesite una notificación pronta al paciente, una evaluación clínica o iniciar un tratamiento, como por ejemplo la TBC, necesitan de comunicación directa.

Es recomendable la documentación de la comunicación establecida o de sus intentos y cualquier discrepancia entre un informe preliminar o urgente y el informe final debería ser comunicado rápida y directamente al médico tratante.

Los ejemplos anteriores forman parte del estándar. Sin lugar a dudas numerosos sucesos requieren comunicación personal con el médico tratante y no están incluidos en el estándar. En estas situaciones prima el buen juicio, el sentido común y el profesionalismo. Ejemplos de esto hay varios:

1. El diagnóstico radiológico efectuado necesita decisiones de manejo terapéutico inmediatas: sonda de alimentación en bronquio, drenaje pleural desplazado, filtración de unión esófago gástrica post quirúrgica, etc.

2. Diagnósticos que constituyen una amenaza potencial a la vida del paciente: cáncer de mama, pulmón, colon.

3. Hallazgos incidentales que son potencialmente peligrosos, encontrados durante otros estudios: cardiomegalia importante en el examen de tórax pre-operatorio, de una hernia inguinal por ejemplo.
Los esfuerzos coordinados con el medico tratante tienen como único fin obtener la mejor atención posible y bienestar para el paciente.

A pesar que puedan existir por parte de muchos radiólogos aprensiones debido a que piensan que los estándares incrementan la posibilidad de riesgo médico-legal, los jueces se inclinan a considerarlos clínica y científicamente válidos, no como un cuerpo único, controlador y evaluador de negligencias, sino como un conjunto de guías claras e informativas en la evaluación de las evidencias presentadas en un caso determinado ${ }^{(13,14)}$.

\section{Discusión}

Los países más avanzados en esta materia han elaborado estándares de comunicación para radiología. Así por ejemplo, el ACR reconoce que la "comunicación es un componente crítico del arte y la ciencia médica y es especialmente importante en radiología diagnóstica".

Esto deja claramente establecido que el diagnóstico provisto por un informe escrito no completa la responsabilidad del radiólogo, sino que la transforma en parte del debido proceso de comunicación, que también incluye la hecha en forma directa. Los radiólogos tenemos el deber de comunicarnos del modo más adecuado, el cómo deberían ser entregados los hallazgos radiológicos y su interpretación, dependen de las circunstancias particulares de un caso dado.

El papel de los estándares de comunicación es asistir en estos esfuerzos y asegurar que los pacientes reciban una atención de calidad ${ }^{(13,14)}$.

Cuando se produce un quiebre de la comunicación, el radiólogo no es absuelto necesariamente de la responsabilidad, aún cuando su diagnóstico sea adecuado y se haya seguido y aplicado correctamente los procedimientos normales de informe. Así, la forma como los hallazgos e interpretaciones de un examen radiológico son comunicados, es tan importante para definir y delimitar el deber y calidad de atención médica, como las habilidades y destrezas involucradas en la interpretación de las imágenes.

Las generalizaciones acerca de cómo los hallazgos radiológicos deberían ser informados y a quien, no son posibles por la variedad de situaciones que se pueden presentar. La mejor política debería ser sopesar el resultado que un potencial quiebre en la comunicación pudiera tener para todas las partes involucradas llámense paciente, médico tratante o radiólogo y de acuerdo a ello eliminar las causas potenciales Decidir una conducta apropiada requiere una combinación de juicio profesional y consideración de las consecuencias. La posibilidad que un informe se pierda en un sistema hospitalario sobrecargado y sobrepasado esta latente, por lo que debemos por todos los medios establecer contacto directo con el médico tratante frente a cualquier informe de "naturaleza crítica"(15). 
Ahora bien ¿Quién define que hallazgos son tan importantes que merecen una comunicación directa con el médico tratante? La respuesta a esta interrogante resume la responsabilidad del radiólogo que efectúa o interpreta el examen.

Frente al gran énfasis que se ha dado a esta responsabilidad del radiólogo, cabe plantearnos si en la vida real, donde a veces la comunicación con los colegas es difícil en ambientes de trabajo recargados y sobre exigidos, que es la mayoría de nuestros sitios de atención, cual es la responsabilidad del medico solicitante para averiguar los resultados de los exámenes. Sin duda una mayor o menor responsabilidad en el seguimiento de los estudios solicitados, marca la diferencia en la gran mayoría de los buenos clínicos. El Comité de Estándares para la Comunicación del ACR en sus instancias de actualización y revisión de normas está proponiendo la posible inclusión de una declaración tal como "el médico tratante tiene una responsabilidad igual que el radiólogo en el seguimiento oportuno de los exámenes radiológicos que solicita" ${ }^{(10)}$.

Está por demás decir que esto no exime al radiólogo de su responsabilidad en la comunicación directa, solo la hace compartida con el clínico. Esto nos conduce inevitablemente a reflexionar que los estándares elaborados no deben ser abstractos, arbitrarios ni carentes de aplicabilidad. Muy por el contrario, deben a la vez garantizar una adecuada atención de los pacientes, pero no deben provocar más daño que ayuda a los radiólogos, al formularse estándares a los cuales en la práctica diaria fuera difícil ceñirse o pongan la vara demasiado alta. Ciertamente deben ser aterrizados y ser el reflejo del buen ejercicio de la radiología contemporánea $a^{(11,12,16,17)}$.

El radiólogo debería ser cuidadoso en la comunicación directa con el paciente, salvo cuando se trate de emergencias, debida consideración que es el tratante el quien tiene el "manejo" del paciente y por lo tanto, responsable de informarlo sobre los resultados de sus exámenes, en el contexto de un diagnóstico y una propuesta terapéutica. Las especialidades de apoyo (radiología, anatomía patológica y otras) rara vez alcanzan una relación médico paciente como la de los médicos tratantes, pero esto no los exime de la obligación de comunicar los hallazgos urgentes, inesperados o inusuales al médico tratante de manera oportuna y adecuada a cada caso en particular e incluso al paciente si fuese necesario.

Debemos dejar asentado que esta concepción está tendiendo a cambiar. Actualmente, muchos pacientes piden o quieren que el radiólogo les informe los resultados de sus exámenes y su significado, lo que se da especialmente en el caso de los pacientes oncológicos (Dr. ¿Mi cáncer se ha ramificado a mis huesos?) o en la mamografía (¿microcalcificaciones agrupadas es cáncer Dra.? $)^{(10)}$.

En nuestro medio, aún no ha comenzado un tratamiento estructurado del problema, dejando las soluciones solo en el ámbito individual, lo que no siempre es enteramente satisfactorio.

\section{Resumen y conclusiones}

La comunicación con los médicos tratantes, sin lugar a dudas, es esencial para una adecuada atención de nuestros pacientes. En ocasiones es difícil y consume tiempo, sin embargo, constituye un estándar de buena atención. Estos estándares consideran en general dos partes del problema: la sustancia de la comunicación y la oportunidad de la misma. La primera tiene que ver con la naturaleza de ellos y la segunda con la forma de comunicación más adecuada de dichos hallazgos. Dicho de otra manera, que, cuando, como y a quien comunicamos ${ }^{(13,14)}$.

El radiólogo no solo es responsable de formular un diagnóstico correcto, sino además, de la comunicación oportuna de los resultados a los médicos tratantes cuando a su juicio el caso así lo amerite. Este énfasis en la comunicación no solo mejora la calidad de la atención a los pacientes, que es nuestro objetivo primordial, sino que además reduce el riesgo médico legal.

Cuando la comunicación directa es necesaria, los aparatos de comunicación electrónica (telefonía móvil, contestadores telefónicos, faxes o correos electrónicos) han llegado a ser de uso generalizado. Sin embargo, ellos no deberían ser utilizados en reemplazo del contacto verbal directo, por el riesgo de producir un quiebre en la comunicación y en la confidencialidad de la información; pese a ello, son medios que el radiólogo puede utilizar en forma restringida para representar al clínico la necesidad de una comunicación directa ${ }^{(13)}$.

Un fenómeno interesante para destacar es el impacto que tiene la tecnología en la forma de comunicarse personalmente entre los médicos clínicos y los radiólogos. Así por ejemplo, la implementación de un ambiente PACS amplio en un servicio de radiología, tiende a disminuir el número de consultas personales debido a la mayor accesibilidad a los estudios de imágenes lo que además resulta en una mejoría en el uso del tiempo clínico. Los resultados de estos estudios señalan que los clínicos tienen mayor tendencia a revisar las imágenes de los exámenes que solicitan en un ambiente sin placas que en uno basado en películas. De este modo, los departamentos de radiología que transiten al ambiente sin películas deberían estar preparados para que la comunicación con los clínicos cambie de ser principalmente verbal a ser llevada cada vez más a través de medios electrónicos ${ }^{(18,19)}$.

\section{Comunicación con nuestros pares radiólogos}

Si la comunicación con los clínicos no es fácil, tampoco lo es con nuestros pares. Como principios generales debemos actuar con honestidad, buscando 
siempre el beneficio del paciente nuestro objetivo ultimo, y no basarnos en una reacción defensiva o corporativa.

Siguiendo esta línea de análisis vemos que se nos pueden presentar varias situaciones. La más cotidiana es la segunda opinión, solicitada por un tratante disconforme con el examen efectuado por otro colega, en el que puede haber por ejemplo, un error de omisión diagnóstica evidente. Nuestra actitud debe ser de respeto hacia los actos radiológicos, sin deslizar comentarios sobre el tema. Mejor aun, deberíamos comunicarnos con el radiólogo que informó ese examen y darle a conocer su error, de manera profesional, respetuosa y responsable, para que actúe en consecuencia, sin olvidar que todos estamos expuestos. La mayoría de nosotros agradeceremos tal comunicación.

Una variante ocurre cuando al momento de informar un examen, por ejemplo una mamografía en la que detectamos un cáncer y al compararla con los exámenes anteriores, efectuado e interpretado por otro radiólogo, vemos que el hallazgo estaba ahí, pero no fue informado. ¿Qué hacemos?

Una alternativa es hacer nada, lo que nos evitaría incomodidades, pero puede ser negativo para el pronóstico de la paciente. ¿Otras opciones? Tal vez comunicarnos con el colega que informó el primer examen sería lo indicado, al igual que en el caso anterior. ¿Es suficiente? Tal vez sí, tal vez no. No tenemos certeza que el médico tratante será informado ni el paciente tampoco, pues al hacer eso es altamente probable la interposición de una demanda en los tribunales en contra de quien cometió el error. Por su parte, el tratante espera que el radiólogo que informa actúe en forma profesional y le comunique todo aquello que beneficia o perjudica la atención de su paciente. He aquí entonces que al haber una fuerte connotación ética, la conducta a seguir tiene que ver con nuestras creencias y actitudes ante el ejercicio de la radiología, $\mathrm{y}$ es plenamente individual ${ }^{(20,21)}$.

\section{ANEXO $\mathrm{N}^{\circ}$ 1: Actos comunicacionales para esculpir en bronce: La comunicación cotidiana.}

Caso $\mathbf{N}^{\circ}$ 1: "Luchito, llévale al radiólogo estas placas y dile que informe las fracturas de las apófisis transversas que no vio" (Traumatólogo al auxiliar del Servicio de Urgencia).

"Dice el doctor que el informe está malo y lo manda para que el radiólogo lo corrija" (El auxiliar a la recepcionista de radiología a plena voz frente a los pacientes de la sala de espera).

Caso $\mathbf{N}^{\circ}$ 2: "Señora, llévele usted misma estas radiografías al radiólogo y hable con él porque tiene que corregir su informe".

"Doctor, vengo a hablar con usted porque mis radiografías están mal informadas y mi médico me mandó a hablar con usted o a repetirme el examen en otro lado. Yo no puedo estar pagando otro examen".
Caso N’3: "Dr. me hago este examen porque mi médico dice que tengo colitis grave".

"Señora su médico no sabe nada, lo que Ud. tiene es un megacolon. Tiene que cambiarse de médico para que le hagan un tratamiento bien hecho".

Caso $N^{\circ} 4$ : "Dr. traje las radiografías que me hice en Cumpeo porque mi médico quiere que las compare con las de ahora".

"Señora estas radiografías son un mamarracho y no se las voy ni a mirar".

\section{Bibliografía}

1. Lingard L, Espin S, Whyte $\mathrm{S}$ et al. Communication failures in the operating room: an observational classification of recurrent types and effects. Qual Saf Health Care 2004;13: 330-334.

2. Firth-Cazens J. Commentary. Why communications fails in the operating room. Qual Saf Health Care 2004; 13: 327.

3. Coeira E, Tombs U. Communication Behaviors in a Hospital Setting: an observational Study. BMJ 1998; 316: 673-676.

4. Jaddad AR, Delamothe T. What next for electronic communication and health care? BMJ 2004; 328: 1143-1144.

5. García C, Ortega D. Comunicación en el Departamento de Radiología: Radiólogos y pacientes. Rev Chil Radiol 2003; 9: 151-156.

6. Gosbee J. Editorial. Communication among health professionals. BMJ 1998; 316: 642-643.

7. García C, Ortega D. El informe radiológico: "Por sus dichos los conoceréis". Rev Chil Radiol 2001; 7: 65-69.

8. Muroff LR. Reformas en salud y radiología. Rev Chil Radiol 1996; 2: 4-9.

9. Dalla-Palma L, Stacull F, Meduri S, Geitung JT. Relationship between radiologists and clinicians: Results from three surveys. Clin Radiol 2000; 55: 602-605.

10. Berlin L. Communicating Findings of Radiological Examinations: Whither Goest the Radiologist's Duty. AJR 2002; 178: 809-815.

11. Lauttin EM. Letter. Writing, Signing and reading the Radiology Report: Who is Responsible and When? AJR 2001; 177: 247-248.

12. Berlin L. Reply AJR 2001; 177: 247-248.

13. ACR Standards (http://www.acr.org/s_acr/index.asp.) Access: 15/10/2003.

14. CAR Standards of communication (http://www.car.ca/ethics/ index.html). Professional Liability. Medical-legal Issues for Residents in Radiology. Jerome $\mathrm{H}$.

15. Shapiro Ed. ACR Medical-Legal Committee $2^{\text {nd }}$ Edition 1994.

16. Dalinka MK, Letter. Communication, the Big Pocket, and the AC R Standards. AJR 2001; 177: 249.

17. Berlin L. Reply AJR 2001; 177: 249.

18. Reiner B, Siegel E, Flagle $C$ et al. Effects of Filmless Imaging on the Utilisation of Radiologic Services Radiology 2000; 215: 163-167.

19. Reiner B, Siegel E, Protopapas Z, Hooper F, Ghebrekidan $\mathrm{H}$, Scanlon M. Impact of filmless radiology on frequency of clinician consultations with radiologists. AJR 1999; 173: 1169-1172.

20. Medina FJ, Bosch E. Aspectos éticos en la práctica de la radiología. Rev Chil Radiol 1996; 2: 4-5.

21. Berlin L. Malpractice issues in breast imaging. In: Breast Imaging. Categorical Course Syllabus. Ed. Dempsey PJ, Monsees B. ARRS. New Orleans.1999. 